|| Print ISSN: 2589-7837 || Online ISSN: 2581-3935 ||

International Journal of Medical Science and Diagnosis Research (IJMSDR)

Available Online at www.ijmsdr.com

NLM (National Library of Medicine ID: 101738824)

Original Research Article

Volume 6, Issue 1; January: 2022; Page No. 09-19

\title{
Efficacy of Nano Crystallised Calcium Phosphate (NCCP) and Casein Phosphopeptide - Amorphous Calcium Phosphate (CPP-ACP) for Controlling Postoperative Sensitivity After Power Bleaching-A Comparative In-Vivo Study
}

\author{
Dr. Ramesh T ${ }^{1}$, Dr. A. Shilpa ${ }^{2}$, Dr. Sarjeev Singh Yadav ${ }^{3}$, Dr. Kavitha.A ${ }^{4}$, \\ Dr. P.Prathibha ${ }^{5}$, Dr. Zainab Ateeq ${ }^{6}$ \\ ${ }^{1,4}$ Assistant Professors, Conservative Dentistry \& Endodontics, Govt Dental College \& \\ Hospital, Hyderabad, Telangana \\ ${ }^{2}$ Clinician, Conservative Dentistry \& Endodontics, LANS Dental Care, Siricilla \\ ${ }^{3}$ Prof \& HOD, Conservative Dentistry \& Endodontics, Govt Dental College \& Hospital, \\ Hyderabad Telangana \\ ${ }^{5}$ Assistant Professor, Conservative Dentistry \& Endodontics, Sree Balaji Medical College \& \\ Hospital and Research institute. Renigunta \\ ${ }^{6}$ Clinician, Conservative Dentistry \& Endodontics, Evoke Dental Clinic, Hyderabad
}

\section{Conflicts of Interest: Nil}

Corresponding author: Dr. Ramesh T

DOI: https://doi.org/10.32553/ijmsdr.v6i1.893

\section{Abstract:}

Tooth bleaching is one of the most popular cosmetic dental procedures opted by the patients who desire pleasing smile. It is the simplest, least invasive means available to lighten discolored vital teeth. A number of desensitizing agents have been tried in an attempt to counteract bleachingrelated sensitivity. This study was done to compare the effect of two different desensitizing agents for controlling post operative sensitivity after power bleach procedure. Sixty volunteers with mild to moderate dental fluorosis in maxillary anterior teeth, who fulfilled the inclusion and exclusion criteria, were randomly selected for this study. After the bleaching procedurea demo was given to the patient while application of desensitising gel and was instructed to use the gel for 14 days.All the patients were recalled at an interval of $1^{\text {st }}$ day, $3^{\text {rd }}$ day, $5^{\text {th }}$ day, $7^{\text {th }}$ day, and on $14^{\text {th }}$ day to record the post operative sensitivity after power bleach procedure. The observations were analyzed using one way analysis of variance (ANOVA), Tukeys post hoc test. On the basis of VAS results, all the groups showed post operative sensitivity of varied intensity at different intervals. NCCP showed less sensitivity followed by CPP-ACP and Control group.

Keywords: Power Bleaching, Post operative sensitivity, Nano Crystallized Calcium Phosphate, Casein Phosphopeptide-Amorphous Calcium Phosphate 


\section{Introduction:}

Dentin hypersensitivity (DH) is characterized by short or transient sharp pain arising from exposed dentin in response to an array of stimuli-such as thermal, mechanical, osmotic, or chemical substances. ${ }^{[1]}$ The main predisposing factor to Dentin hypersensitivity is exposure of dentin, as a result of loss of enamel and/ or gingival recession. Sensitivity resulting from bleaching is seen most frequently in vital teeth. Various desensitizing agents are been used to decrease the incidence of post operative sensitivity, namely 5\%Potassium Nitrate, $0.454 \%$ Fluoride, 10\% Casein Phosphopeptide-Amorphous Calcium Phosphate, Varnishes, Novamin [3] and these are available in the form of cream, paste, varnish and gel.

Nano Crystallized Calcium Phosphate (NCCP) is a desensitising and demineralizing agent. It contains fluoride and potassium nitrate. Since it's nanometric, the hydroxyapatite penetrates a lot of simply within the dentinal tubules and small fissures in enamel, promoting a highquality seal of the tubules and small fissures, and restores the microstructure of tooth and its chemical composition too.

Casein Phosphopeptide-Amorphous Calcium Phosphate (CPP-ACP) found in cow's milkand it is a derivative of milk protein casein. This reduces the teeth sensitivity by binding to the protein and by the deposition of calcium and phosphate ions that block the dentinal tubules. The purpose of the present study was to assess the efficacy of two different desensitizing agents namely NCCP and CPP-ACP on post operative sensitivity after an in-office vital tooth power bleaching in persons diagnosed with mild to moderate dental fluorosis.

\section{Aim of Study}

To compare the effect of two different desensitizing agents namely NCCP and CPPACP for controlling post operative sensitivity after an in-office vital tooth power bleach procedure.

\section{Patients and Methods}

\section{Methodology:}

\section{Patient Selection Criteria:}

A total of 60 healthy patients irrespective of sex were enrolled in the study. The subjects were selected based on inclusion and exclusion criteria from the regular pool of patients visiting the Department of Conservative Dentistry Endodontics, Government Dental College \& Hospital, Hyderabad, Telangana.

\section{The inclusion criteria included the} following:

- Age group individuals - 20-35 years

- Maxillary anterior teeth

- Deans Indices - Mild to Moderate Dental Fluorosis

- No restorations or carious lesions on the anterior teeth.

- No history of tooth sensitivity

- Positive response on cold testing with Ethyl Chloride spray and Electric pulp testing

- No use of a desensitising agent or desensitising toothpaste from past six months.

The Exclusion criteria included the following:

- Gingivitis and Recession

- Wasting diseases like Attrition, Abrasion, Erosion and Abfraction

- Non vital tooth

- Previous Bleaching procedures

- Fracture tooth

Informed consent was taken from the patients. Complete oral prophylaxis was done two weeks before the tooth bleaching appointment. After isolation with light cure gingival barrier, $35 \%$ hydrogen peroxide was applied on upper anterior teeth to be bleached. Tooth surface was exposed for 15 minutes using $\mathrm{c}$ bright 
accelerator unit. At the end of recommended time bleaching agent was removed and the procedure was repeated twice. Light cure gingival barrier was removed and tooth was rinsed thoroughly.

\section{Grouping was done as follows-}

Group I- Control group - Without Desensitizing Agent

Group II- Gel with NCCP

Group III- Gel with CPP-ACP

A demo was given to the patient while application of desensitising gel, the gel assigned for each patient was applied directly on the labial surface of each tooth and left undisturbed for three minutes. The score was not recorded on the day of procedure i.e., Day 0 .All the patients were recalled at an interval of $1^{\text {st }}$ day, $3^{\text {rd }}$ day, $5^{\text {th }}$ day, $7^{\text {th }}$ day, and on $14^{\text {th }}$ day to record the post operative sensitivity after power bleach procedure.

The observations were analyzed using one way analysis of variance (ANOVA), Tukeys post hoc test.

\section{Results}

After power bleaching, post operative sensitivity was assessed at intervals of day 1 , day 3, day5, day 7 and day 14.Patient's were handed out VAS charts and were asked to mark a score correlating to their post operative sensitivity.

\section{On day 1}

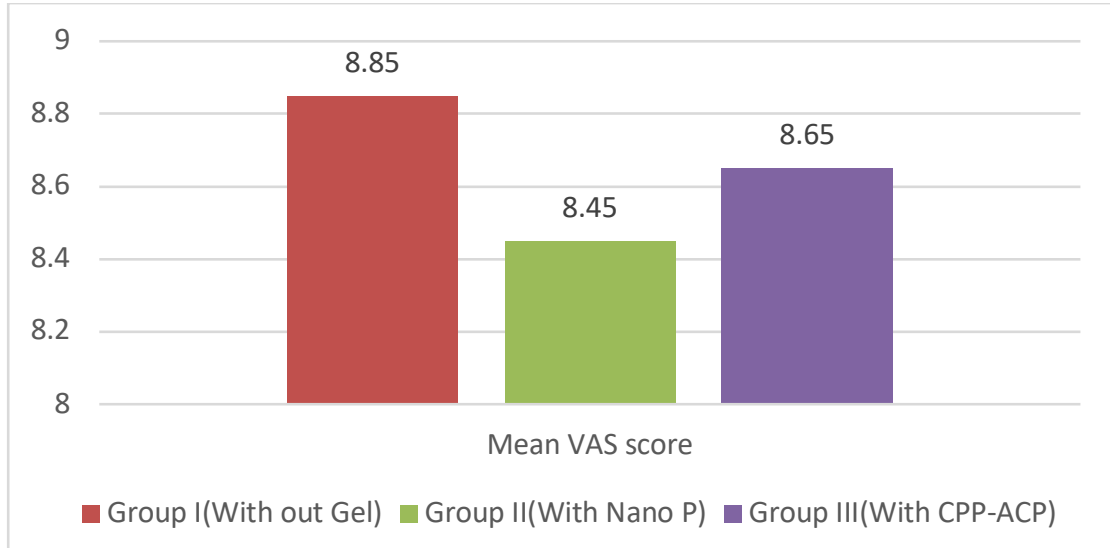

GRAPH I: Comparison of VAS scores on day 1 of three groups

There was no statistically significant difference between the groups

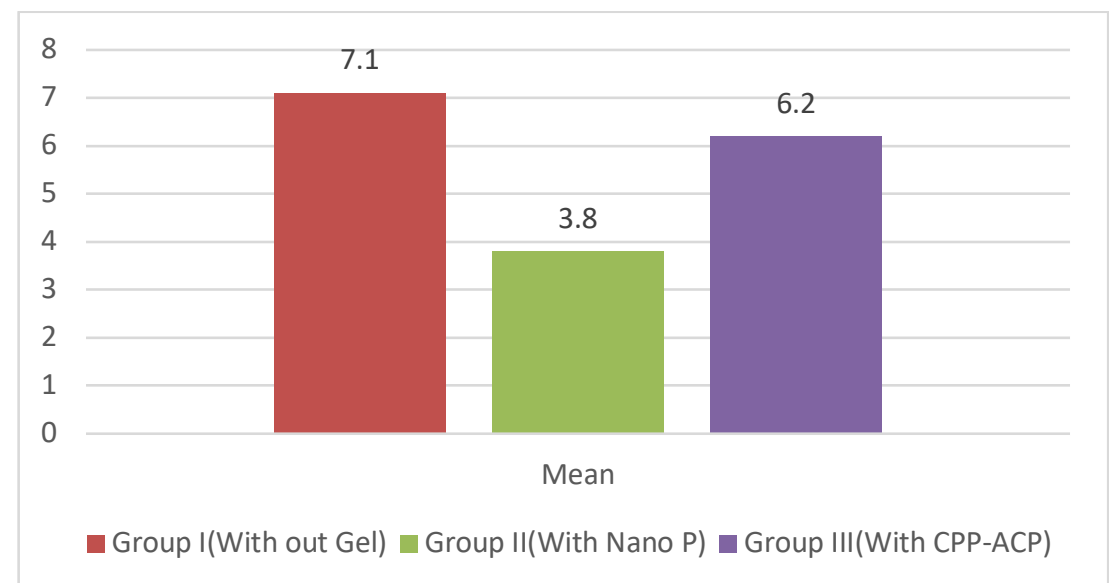

GRAPH II: Comparison of VAS scores on day 3 of three groups 


\section{On day 3}

Group I had shown higher VAS score when compared to group II \& Group III.

Group II had shown lower VAS score when compared to group III.

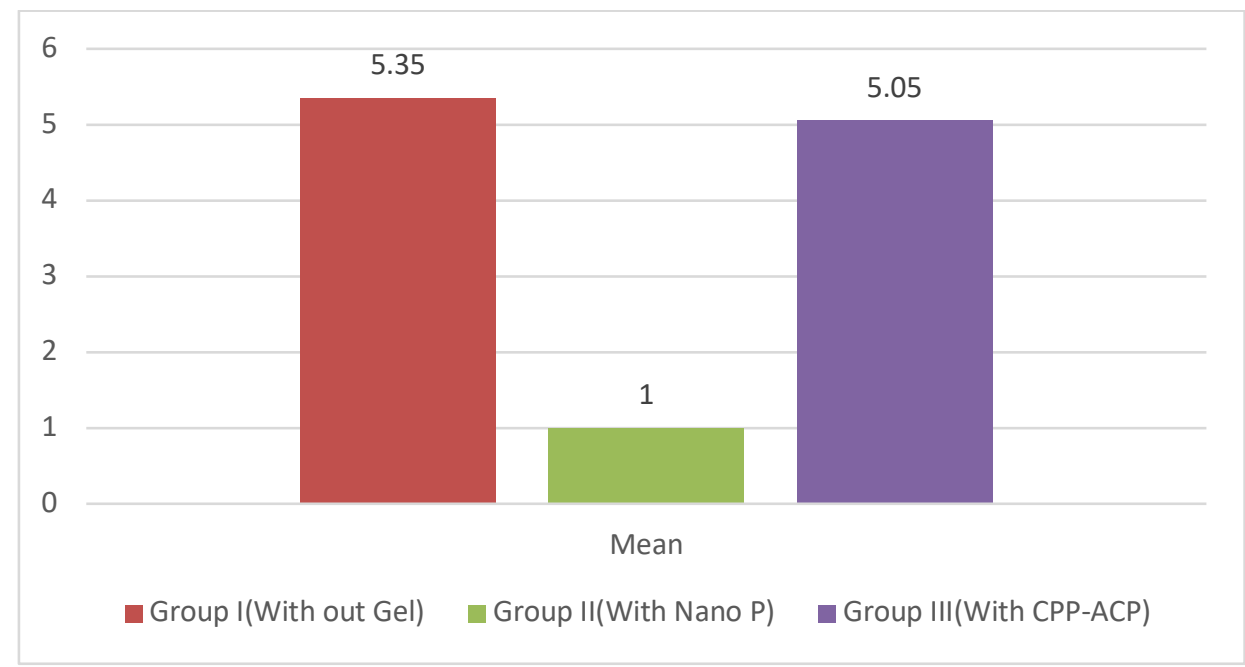

GRAPH III: Comparison of VAS scores on day 5 of three groups

\section{On day 5}

Group I had shown higher VAS score when compared to group II.

Group I had no statistical significant difference when compared to group III.

Group II had shown lower VAS score when compared to group III.

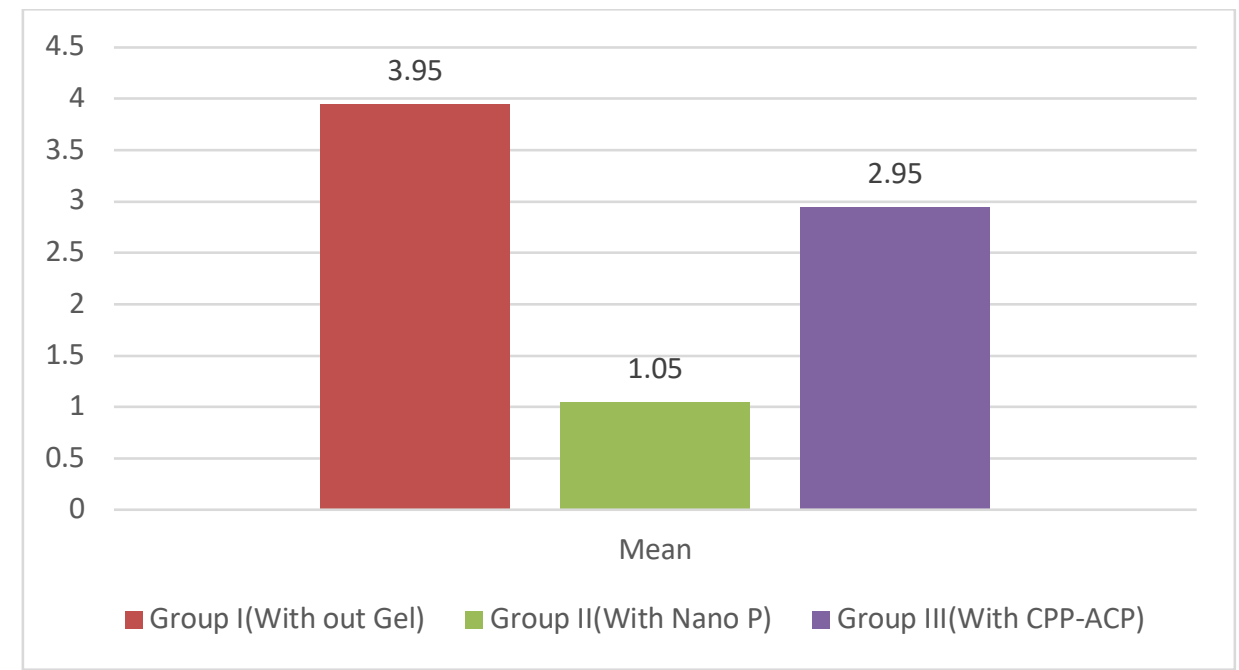

GRAPH IV: Comparison of VAS scores on day 7 of three groups

\section{On day 7}

Group I had shown higher VAS score when compared to group II.

Group I had no statistical significant difference when compared to group III.

Group II had shown lower VAS score when compared to group III. 


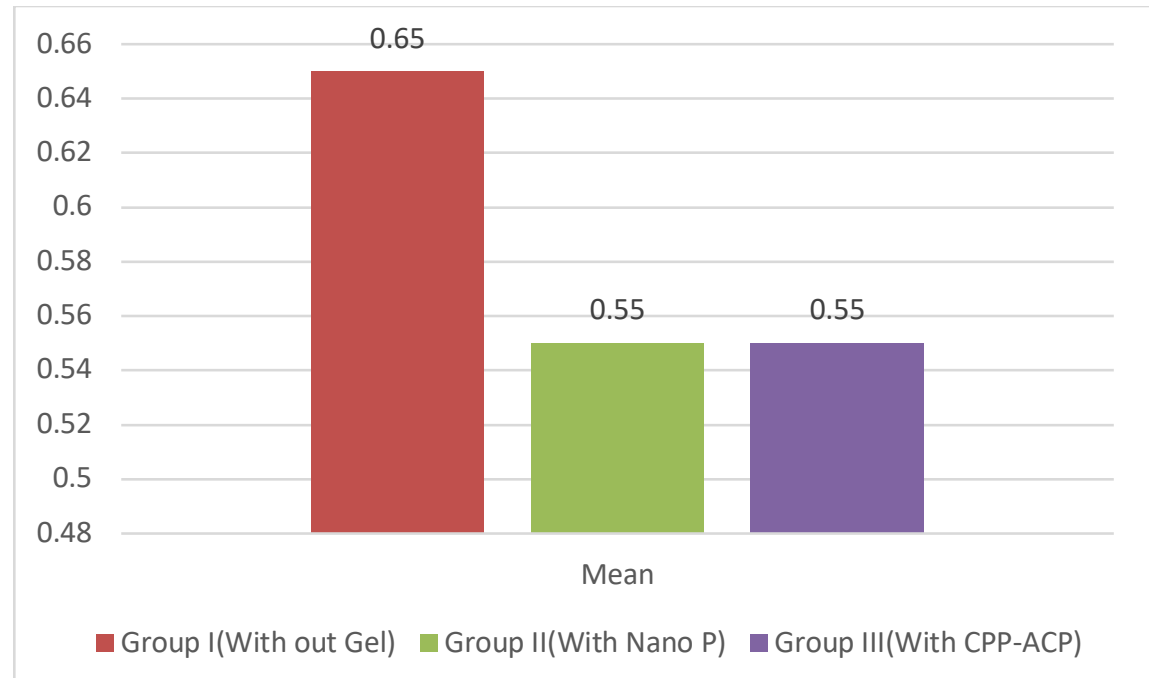

GRAPH V: Comparison of VAS scores on day 14 of three groups

\section{On day 14}

There was no statistically significant difference between the groups.

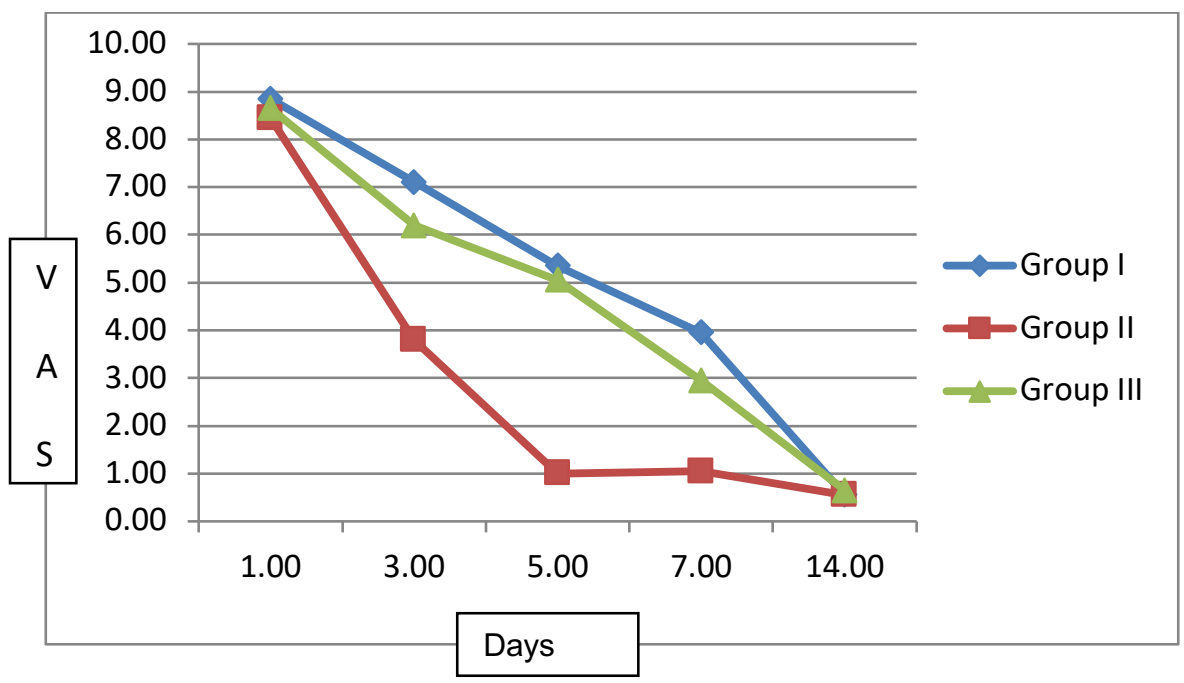

Group I had significantly highest mean sensitivity score than group II \& group III at all intervals. Group III also had significantly lower mean pain score than group I and higher than group II. Least amount of mean pain scores were recorded in group II at all intervals.

\section{Discussion}

Tooth discoloration is commonly associated with clinical and esthetics which is of great concern and it may be intrinsic or extrinsic in nature. Tooth discoloration that occur due to dental fluorosis is a developmental disturbance of enamel caused by excessive exposure to high concentrations of fluoride ( $>1-2$ PPM) during tooth development. Several methods are employed to treat the discolored teeth such as bleaching, micro abrasion; macro abrasion; and restorative procedures such as porcelain veneers, crowns, or composite bonding. ${ }^{[2]}$ Tooth bleaching is one of the most popular esthetic dental procedures for patients who desire pleasing smile. ${ }^{[3]}$ 
Materials, generally used for in-office vital tooth bleaching are hydrogen peroxide and carbamide peroxide. ${ }^{[4]}$ Among which Hydrogen peroxide is the most commonly used bleaching agent for in-office vital tooth bleaching in a range from $25 \%$ to $38 \% .^{[5]}$

Power bleaching uses light energy which is intended to accelerate the process of bleaching in a dental office. It is usually so rapid that visible results are observed even after a single visit which can be used to motivate the patient to continue treatment. Due to increased activation of a bleaching agent by the thermo catalytic technique there has been a deleterious effect on the tooth structure. ${ }^{[6]}$ Among which tooth bleaching sensitivity is the most frequent condition seen in vital tooth bleaching, [7] which occurs as a generalized hypersensitivity but as a spontaneous, sharp, shooting or tingling pain limited to one or a few teeth.

Tooth sensitivity (TS) probably occurred due to a high concentration of the bleaching gel and the longer duration (35\% HP, for 45 minutes). Another fact that contributed to the increase in TS during the in-office bleaching procedure was the application of light and heat sources, which led to higher pulpal temperature. ${ }^{[8]}$

TS is a temporary side effect that disappears after four days of treatment in most patients, but in some cases can persist for up to 10 days. [9]

In the present clinical study, discolored maxillary anterior teeth were bleached with $35 \%$ HP with three applications for $15 \mathrm{~min}$ each in single visit, according to the method used by Reis et al, ${ }^{[10]}$ who observed a better bleaching efficacy when compared with the single 45-min bleaching time. At first glance, multiple bleaching sessions of a single 15minute application have the problem of increasing the bleaching costs, but this drawback may be outweighed by the reduction in the damage produced in the pulp tissue [11] and the reduction in the risk and intensity of TS that arises from this shorter clinical protocol. In addition, a protocol comprising multiple bleaching sessions of a single 15-minute application may play an important role in pulpdentin stimulation and healing. ${ }^{[12]}$

In the current study, patients reported low gingival irritation probably because it was possible to carefully control contact of the bleaching gel with the gingival margin by using light-cured gingival dams. Top dam was used to isolate the gingival tissues, which is a light-curing resin, which replaces the rubber dam (complete isolation) in some cases where relative gum isolation is sufficient. It is the best gingival barrier due to its excellent sealing power, providing easy removal without irritating the gingival tissues.

The bleaching gel used in the present study is Whiteness HP Maxx is a 35\% HP whitening gel, Presented in two phases, peroxide and thickener. It is a product with high bleaching power which can be activated by light or heat (laser, light curing unit, plasma lamps, etc) as well without external auxiliary source (in this case, the period of gel application is longer). When irradiated with light it converts the light into thermal energy, which accelerates the peroxide process penetration in the dental structure. This accelerates the whitening process. To aid in bleaching process, the product contains a group of special dyes which acts as an absorbent barrier also serving as time indicators (its color alters from intense red to bluish green at the end of the process). Additionally to the tints, the product contains an inorganic load that acts as a barrier and collector of heat waves. The product which is recently manipulated presents a red coloration which changes to green after a certain period. This color transition process usually takes from 6- 10 minutes with light and heat application and up to 15 minutes without such application. For this reason, it is recommended regardless of whether or not the light, allow the product to act for 15 minutes. 
The light source used in this study is C BRIGHT Teeth Whitening accelerator unitto activate the action of the bleaching gel $(35 \%$ HP), uses a strong LED light with a specific wavelength (between 420-490nm) and intensity $>6000 \mathrm{~mW} / \mathrm{cm} 2$ which is of high intensity. The use of high-intensity light is to increase the temperature of hydrogen peroxide and to accelerate the rate of bleaching.

The light source used in this study is made of a matrix of LEDs. Although the temperature increase was not measured, one can suppose depending on previous studies that the LED source produces the less thermal insult during the light activation process; ${ }^{[13]}$ this light source was used in the present study for this reason.

Luk et $\mathbf{a l}^{[8]}$ reported that color change were significantly affected by inter action of the bleaching and light variables, and the application of light significantly improved the whitening efficacy of same bleaching materials. In a study of Browning and Swift ${ }^{[14]}$ of power bleaching it was stated that light sources used in tooth whitening do not generate sufficient heat to damage teeth. They concluded that high concentrations of chemicals are responsible for faster whitening and that light sources are therefore superfluous in the whitening process. He et al ${ }^{[15]}$ reported that a LA system produced better immediate bleaching effects than a non-light system with lower concentrations of HP. When high concentrations of HP (25-35\%) were employed, there was no difference in the immediate bleaching effect or short-term bleaching effect between the LA system and the non-light system.

The bleaching gel applied on the tooth surface for 15 minutes and same protocol was followed twice, three gel applications during an appointment can promote the desired results in a single session, after completion of treatment 60 patients were divided into 3 groups 20 patients in each group-Without desensitizing agent, With NCCP, With CPP-ACP.A pea- sized(250mg) amount of the assigned desensitizing agent was applied on the labial surface of each tooth and left undisturbed for 3 minutes.Each patient was given the assigned gel in an unmarked syringe and was instructed to use the agent in a similar manner twice daily for 14 days.

In this study, post bleaching sensitivity was recorded using VAS. Post bleaching sensitivity was evaluated by relying on patient's response to pain. A VAS chart was employed for measuring the sensitivity. On the recall visit patients were handed out these charts and were asked to mark a score correlating to their post operative sensitivity. TS were recorded using the following criteria: no sensitivity, mild, moderate and severe, to simplify the evaluation. The VAS is a horizontal line containing 10 numbers from 0 to 10 ; zero indicates no sensitivity and 10 indicate the worst sensitivity. Patients are asked to rate their sensitivity along the line that best represents the intensity of their pain. The intensity results were categorized according to the following scale: Score 0 -no sensitivity; Score 1-3 - mild; Score 4-6 - moderate; Score 7-10 - severe;

These scores were marked at an interval of $1^{\text {st }}$ day, $3^{\text {rd }}$ day, $5^{\text {th }}$ day, $7^{\text {th }}$ day, and on $14^{\text {th }}$ day to record the post operative sensitivity after power bleach procedure.

The VAS is more sensitive than other measures, especially those with a limited number of response categories. This different from the study by Zekonis and others, ${ }^{[16]}$ in which the assessment was done in five categories: none, slight, moderate, considerable and severe.

Rapid lightening of tooth shade is the main benefit of in office tooth bleaching, but sometimes, transient and intolerable sensitivity can be a drawback associated with this treatment. To overcome this drawback, different desensitizing agents are used in an attempt to prevent, or at least reduce, the intensity of sensitivity to an acceptable level 
for patients. ${ }^{[17]}$ Desensitizing agents may interfere with the penetration of oxidizing molecule during bleaching procedure as it is a remineralizing agent. Hence, the application of desensitizing agents is considered after the bleaching procedure.

The results of the present study showed thatWithout desensitizing gel (control group) had significantly highest mean sensitivity score than NCCP group and CPP-ACP group at all intervals.

CPP-ACP group also had significantly lower mean pain score than without gel group and higher than NCCP group.

Least amount of mean pain scores were recorded in NCCP group at all intervals.

The difference in the incidence of pain in the current study is also in agreement with the study, where placebo group showed more incidence of post bleaching TS than CPP-ACP group. ${ }^{[18]}$

In NCCP group least amount of sensitivity scores were recorded, this is because Desensibilize Nano $\mathrm{P}$ is desensitizing and remineralizing agent based on the technology of nanostructured calcium phosphate, organized in crystalline form of hydroxyapatite - a mineral that is part of the dental structure. In addition to presenting chemical and structural characteristics similar to those of natural hydroxyapatite, the small diameter of its particles and its morphology increase its surface area, its capacity for hydration and wettability, and its solubility, allowing it to release calcium and phosphate ions to the oral cavity at suitable concentrations and speeds. The effectiveness of the paste is based on its capability to provide ions of calcium, phosphate, and fluoride to the demineralized surface of the tooth, which can be rearranged in the form of hydroxyapatite, fluorapatite, or calcium fluoride, with acid resistance similar to that of the natural tooth. In addition to remineralization, the desensitizing effect is based in the capacity that the hydroxyapatite layer has to occlude the dentinal tubules, as well as the effect of depolarizing nerve fibers due to the potassium nitrate. Basic composition: Active ingredients: Nanometersized calcium phosphates (in the form of hydroxyapatite), calcium fluoride, and potassium nitrate. Inactive ingredients: Distilled water, surfactant, thickener, flavouring, sweetener and preservatives. Desensitizing components are fluoride and potassium nitrate in its composition.

There are two mechanisms of action: It contains 9000 PPM of fluoride which inhibits the cariogenic activity of the bacteria, prevents demineralization, and enables desensitisation. The presence of $5 \%$ potassium nitrate enables desensitisation through depolarization of the nerve fibers. Since it's nanometric, the hydroxyapatite penetrates a lot of simply within the dentinal tubules and small fissures in enamel, promoting a high- quality seal of the tubules and small fissures, and restores the microstructure of tooth and its chemical composition too.Fluoride application has also been traditionally used to manage sensitivity, and it is thought to act as tubule blocker to control pulpal fluid flow. The primary mechanism of action of fluoride is the occlusion of dentinal tubules or an increase in enamel hardness. ${ }^{[19]}$ The small particle size also contributes to a greater penetration of the product into the tubules and the microfissures on the enamel, which, in combination with its high resistance to dissolution and the similarity of this hydroxyapatite with what is available in the natural tooth; they enhance its desensitizing and remineralising action, providing longevity to the treatment.

Over the years, Potassium nitrate (KNO3) is used as the desensitizing agent to manage TS, with varying reported success. ${ }^{[20]} \mathrm{KNO} 3$ has a calming effect on the nerve by preventing the nerve from repolarising after it has depolarized in a pain cycle. Potassium ions act directly on intradental nerves by raising extracellular 
potassium ion concentration sufficiently to prevent action potential generation by axonal accommodation. Peacock et $\mathbf{a l}^{[21]}$ have shown that a potassium ion concentration above $8 \mathrm{mM}$ to $16 \mathrm{mM}(0.08-0.16 \%$ as $\mathrm{KNO} 3)$ around the axons is needed to sustain nerve depolarization. Kose, et al ${ }^{[22]}$ reported that pre treatment with $5 \% \mathrm{KNO}_{3}$ and $2 \%$ sodium fluoride $(\mathrm{NaF})$ reduced the duration of sensitivity during bleaching treatment - the placebo group had sensitivity on $35 \%$ of the bleaching days, while the desensitizing agent group had sensitivity on $20 \%$ of the bleaching days. Reis, et al ${ }^{[23]}$ assessed tooth sensitivity following the application of $5 \% \mathrm{KNO}_{3}$ and $2 \%$ $\mathrm{NaF}$ prior to LA bleaching. Less severe sensitivity was experienced by $80 \%$ of those in the experimental group. Haywood $^{[24]}$ performed a study in which one group of patients pre brushed with $\mathrm{KNO}_{3}$ toothpaste for 2 weeks before bleaching, while the other group of patient's pre brushed with fluoride toothpaste. The group using the $\mathrm{KNO}_{3}$ toothpaste experienced less sensitivity and more sensitive- free days than the fluoride toothpaste group.

In the present study CPP-ACP group showed less sensitivity when compared to control group this is because CPP-ACP occludes the dentinal tubules.GC Tooth Mousse The CPPACP complex was patented by the University of Melbourne, Australia. It is generally marketed in the USA as MI Paste and MI Paste Plus and outside the USA, the products are marketed as GC Tooth Mousse ${ }^{\mathrm{TM}}$ (India) and GC Tooth Mousse plus ${ }^{\mathrm{TM}}$ (India). ${ }^{[25]}$ It is a waterbased crème containing RECALDENT (CPP-ACP). When CPP-ACP is applied in the oral environment, it will bind to bio films, plaque, bacteria, hydroxyapatite and soft tissue localising bio-available calcium and phosphate.

As CPP-ACP has the ability to localize ACP at the tooth structure, increasing the level of calcium phosphate in plaque and hence may act as a calcium phosphate reservoir, buffering the free calcium and phosphate ion activities, thereby helping to maintain a state of super saturation with respect to tooth enamel thereby it decreases enamel demineralization and enhances enamel remineralization. ${ }^{[26]}$

Casein phosphopeptide are multi phosphorylated from an enzymatic digest of the bovine milk protein casein. These peptides have remarkable ability to stabilize the calcium phosphate in solution as amorphous calcium phosphate (ACP), through their multi phosphoryl residues CPP bind to forming nanoclusters of ACP in metastable solution, preventing their growth to critical size required for nucleation and phase transformation. CPPACP nanoclusters have shown to localize at the tooth surface, which buffers free calcium and phosphate ion activities by helping to maintain a state of super saturation with respect to tooth enamel depressing demineralization and enhancing remineralization. ${ }^{[27]}$

$\mathrm{CPP}-\mathrm{ACP}$, a remineralizing agent is shown to make teeth less sensitive to hot, cold, air pressure, and tactile stimulation when it is applied topically either by dental professionals or by the patient themselves after tooth bleaching, ${ }^{[28]}$ and the present study results are similar to them. It acts by binding to the protein and deposition of calcium and phosphate ions that block the dentinal tubules. CPP-ACP may interfere with the penetration of oxidizing molecule during bleaching procedure as it is a remineralizing agent. [29] Hence, the application of desensitizing agents is considered after the bleaching procedure.

Bayrak, et al [30] found that CPP-ACP significantly increased enamel microhardness following bleaching with $38 \% \mathrm{HP}$, while fluoride gel had no effect on enamel microhardness. Remineralization by ACP was found to be much more effective than that produced by $\mathrm{KNO}_{3}$ or fluoride alone. ${ }^{31]}$

Results of this study are in agreement with those of some other studies that show high occurrence of sensitivity after concentrated 
peroxide based bleaching. Maghaireh et al $^{[17]}$ reported that $98 \%$ of participants experienced sensitivity after using concentration of $35 \% \mathrm{H} 2$ $\mathrm{O} 2$ for bleaching. Tang and Millar ${ }^{[20]}$ reported that $85.2 \%$ of their study participants had sensitivity after in office bleaching using 15\% $\mathrm{H} 2 \mathrm{O} 2$. Tay et al ${ }^{[32]}$ used $35 \% \mathrm{H} 2 \mathrm{O} 2$ for vital tooth bleaching and reported that $66.8 \%$ participants experienced TS.

The present study concluded that the NCCP showed less sensitivity when compared to CPP-ACP and control group. There are very few clinical studies done to compare the effect of desensitizing agents on tooth sensitivity following an in-office vital tooth bleaching in case of dental fluorosis. Therefore further studies are required to confirm the results. The use of power bleaching can be further explored using different gel concentrations and application times. Such protocols could reduce the risks of TS while promoting the same bleaching effect and could be used in younger patients.

\section{Conclusions}

Within the limitations of this study, the following conclusions can be drawn:

- Desensitising agents show effective reduction in the incidence of sensitivity after an in- office vital tooth power bleaching.

- Significant decrease in sensitivity was seen with NCCP group followed by CPP-ACP group and Control group.

\section{References}

1. Dowell P, Addy M. Dentine hypersensitivity - a review. Aetiology, symptoms and theories of pain production. J Clin Periodontol 1983;10(4):341-50.

2. Greenwall L. Bleaching Techniques in Restorative Dentistry. London: Martin Dunitz Ltd; 2001. p. 205-9.

3. Zekonis R, Matis BA, Cochran MA, Al Shetri SE, Eckert GJ, Carlson TJ. Clinical evaluation of in-office and at-home bleaching treatments. Oper Dent 2003;28:114-21.

4. Klaric E, Profeta I, Matoševic D, Tarle Z. Postoperative sensitivity after two in-office bleaching methods. Acta Stomatol Croat 2011;45(2):52-8

5. Basting RT, Amaral FL, França FM, Flório FM. Clinical comparative study of the effectiveness of and tooth sensitivity to $10 \%$ and $20 \%$ carbamide peroxide home-use and $35 \%$ and $38 \%$ hydrogen peroxide in-office bleaching materials containing desensitizing agents. Oper Dent 2012;37:464-73.

6. Joiner A. The bleaching of teeth: a review of the literature. J dent 2006;34(7):412-9.

7. Tredwin CJ, Naik S, Lewis NJ, Scully CB. Hydrogen peroxide tooth-whitening (bleaching) products: review of adverse effects and safety issues. $\mathrm{Br}$ Dent $\mathrm{J}$ 2006;200(7):371.

8. Luk K, Tam L, Hubert M. Effect of light energy on peroxide tooth bleaching. J Am Dent Assoc 2004;135(2):194-201.

9. Bernardon JK, Sartori N, Ballarin A, Perdigao J, Lopes GC, \& Baratieri LN,Clinical performance of vital bleaching techniques Oper Dent 2010;35(1) 3-10.

10. Reis A, Tay LY, Herrera DR, Kossatz S, Loguercio AD. Clinical effects of prolonged application time of an in-office bleaching gel.Oper Dent 2011;36:590-6.

11. Soares DG, Basso FG, Hebling J, \& de Souza Costa CA, Concentrations of and application protocols for hydrogen peroxide bleaching gels: Effects on pulp cell viability and whitening efficacy J Dent 2014;42(2) 185-198.

12. Matsui S, Takahashi C, Tsujimoto Y, \& Matsushima K, Stimulatory effects of lowconcentration reactive oxygen species on calcification ability of human dental pulp cells J Endod 2009;35(1) 67-72.

13. Hofmann N, Hugo B \& Klaiber B, Effect of irradiation type (LED or QTH) on photoactivated composite shrinkage strain 
kinetics, temperature rise and hardness Eur J Oral Sci 2002;110(6) 471-479.

14. Browning WD, Swift EJ Jr. Critical appraisal. Power bleaching. J Esthet Restor Dent 2011;23:61-7.

15. He LB, Shao MY, Tan K, Xu X, Li JY. The effects of light on bleaching and tooth sensitivity during in-office vital bleaching: A systematic review and meta-analysis. J Dent 2012;40:644-53.

16. Zekonis R, Matis BA, Cochran MA, Al Shetri SE, Eckert GJ \& Carlson TJ, Clinical evaluation of in-office and at-home bleaching treatments Oper Dent 2003;28(2) 114-121.

17. Maghaireh GA, Alzraikat H, Guidoum A. Assessment of the effect of casein phosphopeptide-amorphous calcium phosphate on postoperative sensitivity associated with in-office vital tooth whitening. Oper Dent 2014;39(3):239-47.

18. Nanjundasetty JK, Ashrafulla M. Efficacy of desensitizing agents on postoperative sensitivity following an in-office vital tooth bleaching: a randomized controlled clinical trial. J Conserv Dent 2016;19(3):207.

19. Najibfard K, Ramalingam K, Chedjieu I, Amaechi BT. Remineralization of early caries by a nano-hydroxyapatite dentifrice. J Clin Dent 2011;22(5):139.

20. Tang B, Millar BJ. Effect of chewing gum on tooth sensitivity following whitening. Br Dent J 2010;208:571-7.

21. Peacock JM, Orchardson R. Effects of potassium ions on action potential conduction in A- and C-fibers of rat spinal nerves. J Dent Res 1995;74:634-41.

22. Kose C, Reis A, Baratieri LN, Loguercio AD. Clinical effects of at-home bleaching along with desensitizing agent application. Am J Dent 2011;24:379-82.

23. Reis A, Dalanhol AP, Cunha TS, Kossatz $\mathrm{S}$, Loguercio AD. Assessment of tooth sensitivity using a desensitizer before light-activated bleaching. Oper Dent 2011;36:12-7.

24. Haywood VB, Cordero R, Wright K, Gendreau L, Rupp R, Kotler M, et al. Brushing with a potassium nitrate dentifrice to reduce bleaching sensitivity. J Clin Dent 2005;16:17-22.

25. Deepa B, Puranik M P, Uma S R . Casein phosphopeptide - Amorphous calcium phosphate: A review. Int J Dent Health Sci 2015;2:116-25.

26. Gagnaire V, Pierre A, Molle D, Leonil J. Phosphopeptides interacting with colloidal calcium phosphate isolated by tryptic hydrolysis of bovine casein micelles. J Dairy Res 1996;63:405-22.

27. Reynolds EC. Anticariogenic complexes of amorphous calcium phosphate stabilized by casein phosphopeptides: A review. Spec Care Dentist 1998;18:8-16.

28. Ginger M, MacDonald J, Ziemba S, Felix H. The clinical performance of professionally dispensed bleaching gel with added amorphous calcium phosphate. J Am Dent Assoc 2005;136:383-92.

29. Gillen T, Carr MP, Rashid R. The effect of CPP-ACP on sensitivity associated with tooth whitening. Journal of Ohio State University, Columbus, OH. 2008.

30. Bayrak S, Tunc ES, Sonmez IS, Egilmez T, Ozmen B. Effects of casein phosphopeptide-amorphous calcium phosphate (CPP-ACP) application on enamel microhardness after bleaching. Am J Dent 2009;22:393-6.

31. Tschoppe P, Neumann K, Mueller J, Kielbassa AM. Effect of fluoridated bleaching gels on the remineralization of predemineralized bovine enamel in vitro. $\mathrm{J}$ Dent 2009;37:156-62.

32. Tay LY, Kose C, Loguercio AD, Reis A. Assessing the effect of a desensitizing agent used before in-office tooth bleaching. J Am Dent Assoc 2009;140:1245-51. 